\title{
Des atomes ultrafroids sous le microscope
}

Thierry Lahaye ${ }^{(1)}$ (lahaye@irsamc.ups-tlse.fr) et Stefan Kuhr ${ }^{(2)}$ (stefan.kuhr@strath.ac.uk)

(1) Laboratoire Collisions, Agrégats, Réactivité, CNRS UMR 5589 et Université Paul Sabatier, 118 route de Narbonne,

31062 Toulouse Cedex 4.

(2) Max-Planck-Institut für Quantenoptik, Garching, Allemagne, et University of Strathclyde, Glasgow, Royaume-Uni.

Des atomes ultrafroids peuvent

être piégés dans un réseau

périodique obtenu à l'aide

de faisceaux laser.

De même que les électrons

dans un solide, les atomes

dans un tel « réseau optique »

peuvent se comporter comme

un métal supraconducteur ou

au contraire comme un isolant.

Les physiciens savent désormais

observer ces phénomènes au

microscope, atome par atome,

avec une résolution comparable

au pas du réseau.

Ils peuvent aussi manipuler

sélectivement les atomes

d'un site donné, en changeant

leur état quantique. Ces pro-

grès ouvrent des perspectives

enthousiasmantes pour

le traitement quantique

de l'information.
De nombreux phénomènes physiques intéressants, tels que la supraconductivité à haute température critique, résultent des interactions fortes existant entre les particules dans un système quantique à $\mathrm{N}$ corps. Une perspective alléchante pour les physiciens est de simuler le comportement de ces systèmes à l'aide de nuages d'atomes froids placés dans des réseaux optiques [1], les atomes jouant ici le rôle des électrons dans le réseau cristallin.

\section{Les réseaux optiques}

L'avantage de ce type de simulation est que ces réseaux optiques sont presque exempts de défauts. Par ailleurs, les interactions entre les particules individuelles peuvent être modifiées à volonté par l'expérimentateur. Jusqu'à très récemment, dans les réseaux optiques, les physiciens ne pouvaient mesurer que des densités atomiques moyennes sur plusieurs sites, tandis que les variables locales, telle que la répartition exacte des particules sur le réseau, étaient inaccessibles. Les travaux récemment publiés par les groupes de Stefan Kuhr et Immanuel Bloch, au Max-Planck-Institute for Quantum Optics à Garching, et de Markus Greiner, à Harvard [2, 3], remédient à cette lacune. Ces physiciens sont ainsi parvenus, pour la première fois, à observer et à manipuler des atomes individuels dans un système à $\mathrm{N}$ corps au sein d'un réseau optique.

Pour charger des atomes de rubidium dans un tel réseau, il faut d'abord les refroidir à des températures de quelques milliardièmes de degré au-dessus du zéro absolu. Le réseau optique est obtenu en superposant plusieurs faisceaux laser, ce qui donne naissance à une figure d'interférences où alternent zones éclairées et

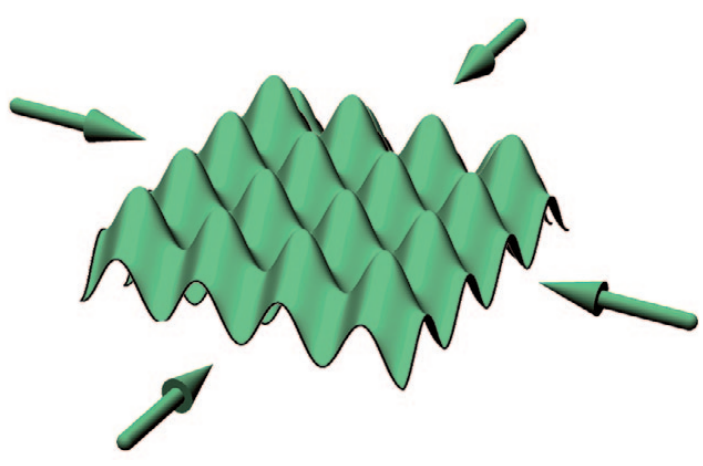

1. Obtention d'un réseau optique bidimensionnel (potentiel périodique au centre, en forme de « boîte à œufs »), grâce à quatre faisceaux laser (symbolisés par les flèches). En augmentant la profondeur du réseau optique, on diminue (exponentiellement) le passage par effet tunnel des atomes d'un site du réseau à l'autre, et on passe ainsi du régime superfluide au régime isolant de Mott.

zones sombres, disposées en damier. Les atomes sont attirés vers les régions où l'intensité de la lumière est maximale, et sont ainsi piégés sur ces sites du réseau optique, comme le seraient des billes dans les creux d'une boîte à œufs (fig. 1).

\section{Voir des atomes individuels}

Afin d'observer les atomes, les chercheurs ont mis en place un microscope optique spécialement conçu, avec une très grande ouverture numérique (fig. 2). Dans le réseau, les atomes sont éclairés en permanence par des faisceaux laser qui permettent de les refroidir ; ils diffusent ainsi de la lumière de fluorescence qui est recueillie par l'objectif du microscope. À la longueur d'onde utilisée, qui est de $780 \mathrm{~nm}$, la résolution est d'environ $700 \mathrm{~nm}$, soit à peine plus que la distance entre les atomes dans le réseau (ici $532 \mathrm{~nm}$ ), ce qui est suffisant pour identifier sans ambiguïté des atomes situés sur des sites voisins du réseau. En utilisant une caméra CCD spéciale, on peut détecter environ 5000 photons diffusés par 


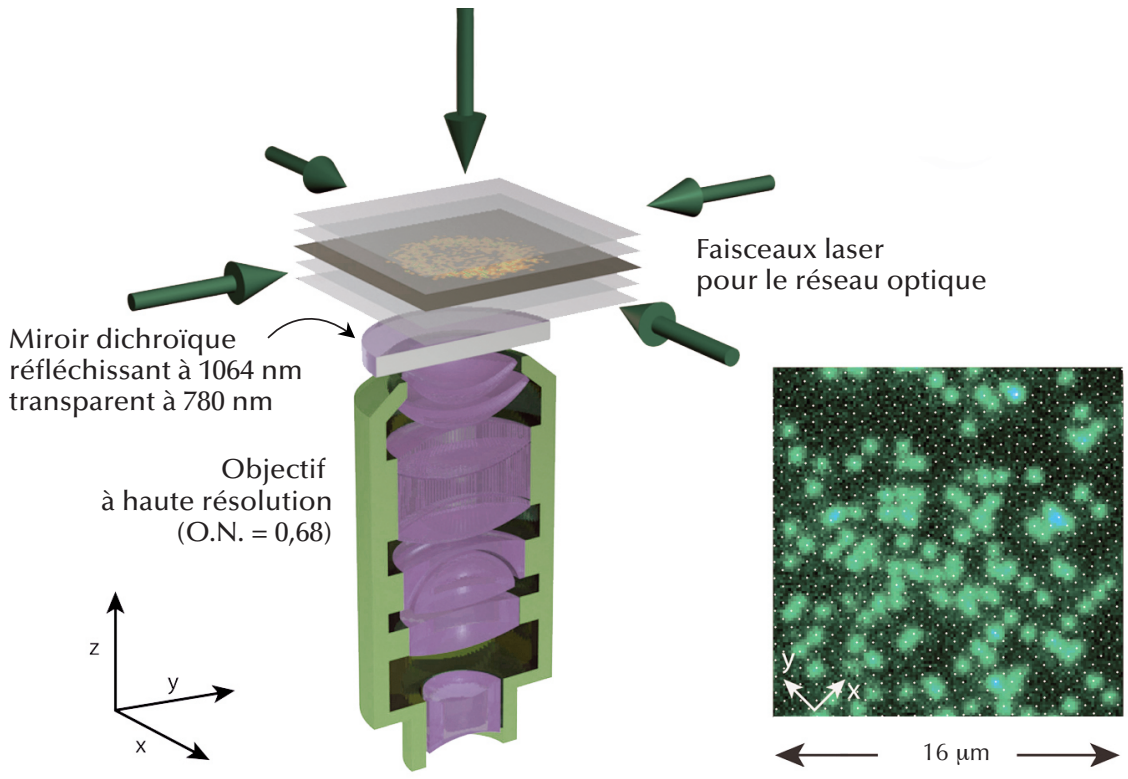

2. Représentation schématique du dispositif expérimental [3]. Les atomes sont préparés dans un plan du réseau optique (longueur d'onde $1064 \mathrm{~nm}$ ), et observés à l'aide d'un objectif de microscope à haute résolution (ouverture numérique de 0,68). En bas à droite : image par fluorescence d’atomes uniques dans le réseau optique. La distance entre sites du réseau (indiqués par des points blancs) vaut $532 \mathrm{~nm}$.
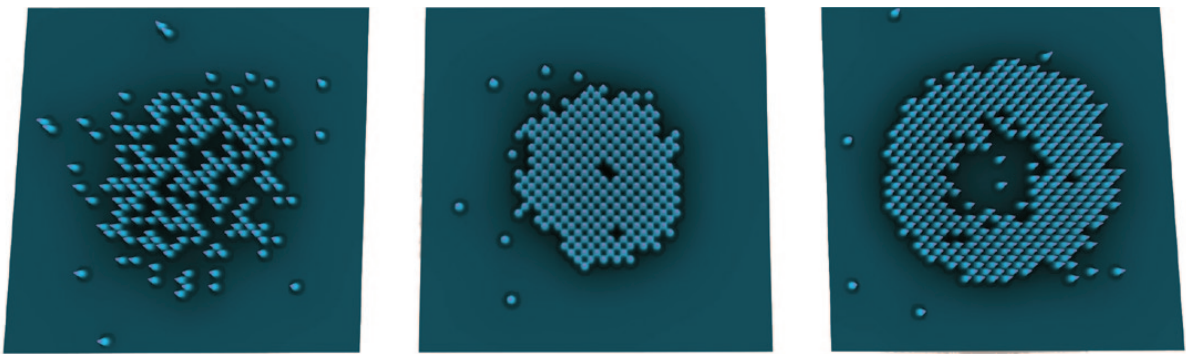

3. Atomes dans le réseau optique. Dans un condensat de Bose-Einstein (superfluide, à gauche), les atomes sont délocalisés sur tout le réseau, et le nombre d’atomes par site présente des fluctuations importantes. Dans un isolant de Mott (au centre et à droite), les atomes s'organisent en coquilles concentriques contenant un nombre bien déterminé d’atomes par site (deux atomes par site au centre du piège, un atome par site en périphérie).
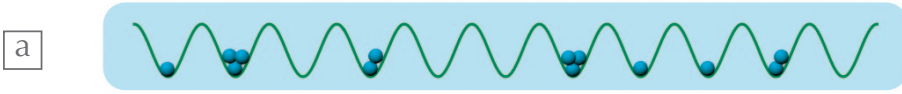

$\mathrm{b}$
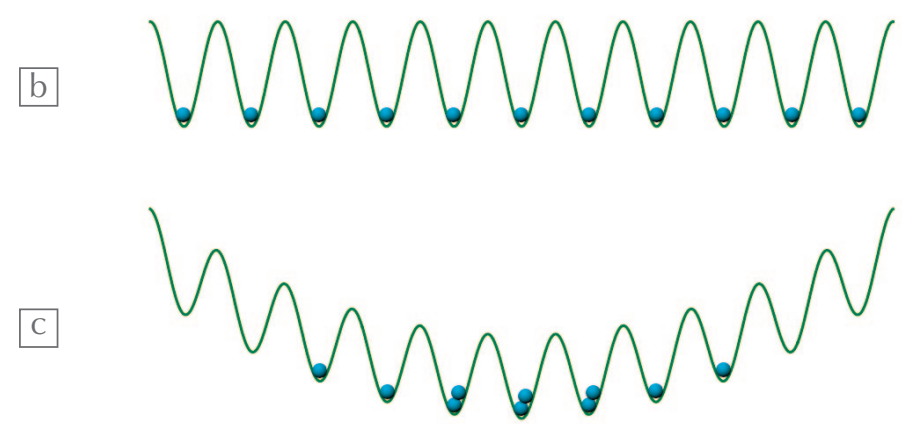

4. Représentation schématique des atomes piégés sur les sites du réseau optique.

a : phase superfluide, où les atomes sont délocalisés sur tout le réseau, et où le nombre d'atomes par site présente donc de fortes fluctuations.

b : phase « isolant de Mott », où l'énergie de répulsion entre atomes empêche deux atomes de se retrouver sur le même site (on a donc ici un et un seul atome par site).

c : en réalité, un potentiel harmonique se superpose au réseau optique, et la configuration minimisant l'énergie totale est ici un isolant de Mott à deux atomes par site au centre du nuage, et à un atome par site en périphérie (voir texte).

\section{\〉}

atome, et ainsi voir distinctement sur les images les atomes individuels. Si plusieurs atomes se trouvent initialement sur un même site du réseau, la lumière laser induit des collisions inélastiques entre les atomes, et l'énergie relâchée est suffisante pour les faire sortir (par paires) du réseau. Ainsi, si le nombre initial d'atomes sur un site est impair, on observe in fine un atome sur ce site. Dans le cas où le nombre initial d'atomes est pair, on observe au contraire un site vide.

\section{La phase « isolant de Mott »}

Les images ainsi obtenues (fig. 3) montrent très clairement ce qui n'avait été jusqu'à présent mesuré que de manière indirecte, à savoir la transition de phase quantique d'un superfluide à un isolant de Mott [4], du nom du prix Nobel britannique Sir Nevill F. Mott. Dans un superfluide, les atomes forment un condensat de BoseEinstein et sont délocalisés sur tout le réseau. On peut montrer qu'alors, le nombre de particules par site du réseau présente des fluctuations importantes (fig. 4a). Dans un isolant de Mott, au contraire, les particules restent "bloquées » sur leur site, car les interactions répulsives entre atomes suppriment le passage par effet tunnel des atomes d'un site du réseau à l'autre. Dans ce cas, le nombre d'atomes sur chaque site $\mathrm{du}$ réseau est bien déterminé (fig. 4b). Cependant, en raison du principe d'incertitude de Heisenberg, la phase des ondes de matière est alors complètement indéterminée, de sorte que les particules ne peuvent désormais plus former un condensat de Bose-Einstein.

Dans la phase isolant de Mott, les atomes ne sont pas distribués de façon uniforme au sein du nuage, qui est circulaire, mais sous forme de quelques " coquilles " concentriques. Ceci est dû au profil d'intensité gaussien des faisceaux laser utilisés pour créer le réseau optique, qui entraîne que les sites situés à la périphérie du réseau ont une énergie plus élevée que celles des sites centraux (fig. 4c) : un potentiel harmonique se superpose au réseau. Bien entendu, lorsqu'un site du réseau est occupé par deux atomes, cela coûte aussi de l'énergie, du fait de l'interaction répulsive entre les deux atomes. Lorsqu'on augmente le nombre d'atomes dans le système, l'état de plus basse énergie globale est donc d'abord obtenu en remplissant chaque site du réseau (en partant du centre) avec un 
seul atome (fig. 3, panneau central), et ce jusqu'à ce que le décalage d'énergie dû au piège harmonique ne devienne plus grand que l'énergie d'interaction de deux atomes occupant un même site. Il devient alors avantageux, sur le plan énergétique, d'avoir des sites doublement occupés au centre du réseau, et des sites occupés par un seul atome en périphérie (fig. 3, panneau de droite, et fig. 4c). C'est ce mécanisme qui conduit à la formation de cette structure en couches (ici, deux), caractéristique de l'isolant de Mott. Cette expérience a en outre permis d'observer directement, pour la première fois, les défauts qui existent dans une telle structure (par exemple, on a, dans la couche à un atome par site, des lacunes et des sites doublement occupés), et d'étudier comment le nombre de défauts augmente avec la température.

\section{Manipuler les atomes un par un}

Dans une autre expérience, dont les résultats ont été récemment publiés [5], les chercheurs ont réussi à manipuler individuellement les atomes, site par site, en changeant leur état interne de spin. En utilisant encore une fois l'objectif à haute résolution, un faisceau laser est focalisé sur un spot de $600 \mathrm{~nm}$ de diamètre, limité par diffraction. À l'aide d'un miroir commandé par des cales piézoélectriques, on peut déplacer ce faisceau laser sur la totalité du champ de l'image, et donc éclairer de manière sélective un ou plusieurs atomes du réseau. Un atome éclairé par ce faisceau voit la différence d'énergie entre ses états internes se modifier légèrement. En soumettant maintenant l'ensemble des atomes à une impulsion micro-onde bien choisie, on induit un basculement du spin uniquement pour les atomes éclairés par le faisceau laser. Les autres atomes du réseau ne sont pas affectés par le champ micro-onde, avec lequel ils ne sont pas à résonance, et restent donc dans leur état de spin initial.

Afin d'estimer de façon quantitative la qualité de cette nouvelle technique d'adressage, les chercheurs ont tout d'abord créé un isolant de Mott avec exactement un atome par site. Puis, ils ont fait basculer le spin de tous les atomes situés sur des sites dessinant une figure particulière (par exemple un carré, comme sur la figure 5 à gauche). Par la suite, ces atomes sont expulsés du réseau à l'aide d'un faisceau laser résonant, et on ne conserve que les atomes dont le spin n'a pas été basculé. On ne voit donc sur
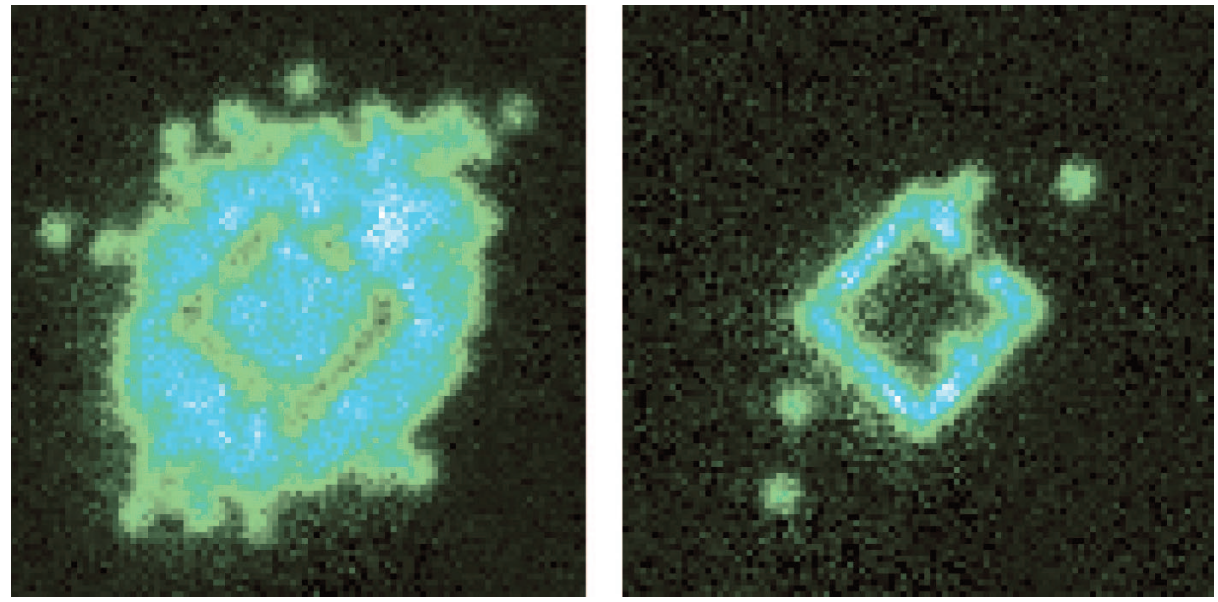

5. Adressage de sites individuels du réseau [5]. Dans un isolant de Mott (à gauche), on renverse les spins des atomes situés le long d'un carré de $7 \times 7$ sites. Les atomes dont le spin a été renversé sont ensuite retirés à l'aide d'un faisceau laser. Dans le cadre de droite, une rotation globale du spin de tous les atomes permet de ne garder que ceux qui ont été adressés.

les images que les atomes non manipulés. L'analyse des images expérimentales a montré que l'adressage fonctionne avec une probabilité très élevée, de $95 \%$. En outre, les atomes situés sur les sites voisins des sites manipulés ne sont pas influencés par le faisceau d'adressage. De même, les physiciens ont réussi à démontrer que la résolution d'adressage est encore meilleure que la limite de diffraction du faisceau laser. Ceci est simplement dû au fait que le déplacement d'énergie induit par le faisceau laser d'adressage est plus grand quand les atomes se trouvent exactement au centre de celui-ci.

Ce nouvel outil permet de créer des distributions arbitraires d'atomes dans le réseau. Par exemple, on peut préparer 16 atomes alignés sur des sites voisins du réseau, et observer ensuite comment ils évoluent en passant à d'autres sites voisins par effet tunnel. En effet, selon les lois de la mécanique quantique, les atomes peuvent se déplacer d'un site du réseau à l'autre, même si leur énergie est insuffisante pour passer classiquement au-dessus de la barrière de potentiel qui sépare deux sites. Ce comportement purement quantique, à l'origine de nombreux effets physiques bien connus, peut ici, pour la première fois, être observé directement à l'échelle de l'atome unique.

\section{Un futur ordinateur quantique?}

Ces expériences ouvrent de nombreuses perspectives intéressantes pour étudier la dynamique collective d'états quantiques à
$\mathrm{N}$ corps. Par exemple, après avoir retiré de manière contrôlée un atome en un point du réseau, on pourrait étudier la façon dont cette perturbation se propage dans le système. Les atomes disposés régulièrement dans le réseau optique pourraient aussi servir de qubits ${ }^{(1)}$ dans un futur ordinateur quantique : en utilisant la technique d'adressage individuel, on peut coder et lire individuellement les qubits. Pour construire des circuits quantiques, il reste encore à pouvoir réaliser des opérations logiques élémentaires entre deux atomes arbitraires dans le réseau. Démontrer une telle porte quantique à deux qubits dans ce système est aujourd'hui l'objet de recherches très actives.

(1) On appelle " qubit " un état quantique constitué d'une superposition linéaire de deux états de base, par convention nommés $\mid 0>$ et $\mid 1>$, et qui représente la plus petite unité de stockage d'information quantique.

\section{Références}

1•I. Bloch, J. Dalibard et W. Zwerger, "Many-body physics with ultracold gases", Reviews of Modern Physics $\mathbf{8 0}$ (2008) 885

2•W. Bakr et al., "Probing the Superfluid-to-Mott Insulator Transition at the Single-Atom Level", Science 329 (2010) 547.

3• J. F. Sherson et al., "Single-atom-resolved fluorescence imaging of an atomic Mott insulator", Nature $\mathbf{4 6 7}$ (2010) 68.

4• M. Greiner et al., "Quantum phase transition from a superfluid to a Mott insulator in a gas of ultracold atoms", Nature 415 (2002) 39.

$5 \bullet$ C. Weitenberg et al., "Single-spin addressing in an atomic Mott insulator", Nature 471 (2011) 319. 